\title{
Kähler-Einstein Metrics on Kummer Threefold and Special Lagrangian Tori
}

PENG LU

\section{Introduction.}

The concept of special Lagrangian submanifolds is introduced by Harvey and Lawson in the seminal paper [HL]. In [SYZ], Strominger, Yau and Zaslow propose a construction of the mirror threefold $Z$ of a given Calabi-Yau threefold $X$ using special Lagrangian tori. Roughly speaking, the mirror threefold $Z$ should be realized as the compactified moduli space of special Lagrangian 3-torus $T$ on $X$ together with flat $U(1)$-connections on $T$. In particular, if the mirror threefold of $X$ exists, then $X$ should admit a fibration with general fiber being a special Lagrangian 3-torus.

Recall that a Calabi-Yau manifold is a compact connected Kähler manifold with vanishing first Chern class. By Yau's theorem ([Y]), given a Calabi-Yau manifold $X$ there exists an unique Ricci-flat metric $\omega$ in the Kähler class (Here we have identified a Kähler metric with its associated Kähler form). Note that a Ricci-flat Kähler metric is equivalent to a Riemannian metric whose holonomy group is a subgroup of $S U(n)$. This metric is the so-called Calabi-Yau metric. Now we recall the notion of special Lagrangian submanifolds.

Definition 1.1. Let $X$ be a Calabi-Yau manifold of complex dimension $n$ with a Calabi-Yau metric $\omega$ and a holomorphic $n$-form $\Omega$ normalized in the following sense that

$$
(-1)^{n(n-1) / 2}(\sqrt{-1} / 2)^{n} \Omega \wedge \bar{\Omega}=\omega^{n} / n ! .
$$

Note that $\Omega$ is unique up to a phase factor $e^{i \theta}, \theta \in \mathbb{R}$. We will call $(X, \omega, \Omega)$ a Calabi-Yau triple. A special Lagrangian submanifold of $(X, \omega, \Omega)$ is a compact manifold $M$ of real dimension n together with an immersion $f$ : $M \rightarrow X$ such that

$$
f^{*} \omega=0 \text { and } f^{*} \operatorname{Im}\left(e^{i \theta} \Omega\right)=0 \text { for some } \quad \theta \in \mathbb{R} .
$$


Given a Calabi-Yau triple $(X, \omega, \Omega)$ the deformation theory of a special Lagrangian submanifold $f: M \rightarrow X$ is studied by McLean ([M]). His result says that the infinitesimal deformation space can be identified with $H^{1}(M, \mathbb{R})$ and every infinitesimal deformation can be realized as an actual deformation.

In section 2, we will study how the special Lagrangian submanifold deforms when the Calabi-Yau metric varies (keeping the $S U(n)$ holonomy). Note that deforming the Calabi-Yau metric is equivalent to deforming both the Kähler class and the complex structure on $X$ by Yau's theorem. Therefore we can separate our deformation into deforming the Kähler class and deforming the complex structure on $X$. We prove that the deformation of $f$ is unobstructed when the complex structure on $X$ varies and we also identify the obstruction when the Kähler class varies.

In section 3 we study the degeneration of Calabi-Yau metrics on Kummer threefold $Y$. Here $Y$ is obtained as the minimal resolution of the quotient $Y_{0}$ of the product of three elliptic curves by a diagonal $\mathbb{Z}_{3}$ action. We glue the complete Calabi metric ([Ca]) on each of the neighborhoods of the exceptional fibers to the background flat metric. We show that they are approximate Calabi-Yau metrics on $Y$. In $K 3$ surface case this phenomenon was studied by Page $([\mathrm{P}])$, Kobayashi $([\mathrm{K}])$ and others. The main point is certain $C^{k, \alpha}$ estimates which are uniform in gluing parameters. For the crucial $C^{0}$ estimate, we need a lower bound on Green's function in terms of geometric data (see Lemma 3.3). The proof of the lemma is given by Peter $\mathrm{Li}$ and is communicated to us by Naichung C. Leung. We also borrow a trick from $[\mathrm{K}]$ to get the desired $C^{k, \alpha}$ estimate.

Once we have understood the degeneration of Calabi-Yau metric on $Y$, we can use the deformation theory developed in section 2 to obtain special Lagrangian tori on $Y$. Here we use the fact that there are special Lagrangian (immersed) tori in the singular variety $Y_{0}$ with respect to the degenerated Calabi-Yau metric (induced from the flat metric on the elliptic curve).

However the threefold $Y$ is rigid and does not have a mirror in the usual sense. Also the special Lagrangian tori constructed here does not give rise to a fibration. Thus this example can not be used as an example of the SYZ construction.

We remark that it is nontrivial to show the existence of special Lagrangian submanifolds. The few examples we know are: 1)Special Lagrangian torus (singular) fibrations in certain $K 3$ surfaces. In this case, the existence is proved by using the twistor family of complex structures which carries a elliptic fibration with respect to one complex structure to a special Lagrangian torus fibration with respect to another complex struc- 
ture (see [HL], p154 or [GW], for example); 2)Bryant's examples ([B]) of the fixed point sets of anti-holomorphic involutions; 3)Special Lagrangian torus fibrations of Borcea-Voisin threefolds ([GW]) with respect to degenerate Calabi-Yau metrics.

Note that besides Kummer threefold $Y$, there are many Calabi-Yau threefolds which can be obtained as the resolutions of a finite group quotient of Abelian varieties. They are extensively studied by Roan ([R]) and others. To study special Lagrangian submanifolds on these spaces, we need to have a good model of complete Ricci-flat metrics near each of exceptional fibers. For $Y$ as above, the exceptional fiber is $\mathbb{C} P^{2}$ and such metric was constructed explicitly by Calabi ([Ca]).

The author would like to thank Naichung C. Leung and Peter Li for their help and Yu Yuan for useful discussion. Also the author thank the referee for pointing out a mistake in the previous version of this paper.

\section{Deformation of Special Lagrangian Submanifolds.}

Assume that there is a smooth family of complex structure $J_{t}$ on compact connected differential manifold $X, t \in(-\varepsilon, \varepsilon)$. Denote $X$ with complex structure $J_{t}$ by $X_{t}$. Assume that $\left(X_{t}, \omega_{t}, \Omega_{t}\right)$ is a family of Calabi-Yau triples. Let $f_{0}: M \rightarrow X_{0}$ be a special Lagrangian submanifold. We want to study the obstruction to deforming $f_{0}$ as $t$ varies. Let $f_{t}: M \rightarrow X_{t}$ be a family of special Lagrangian submanifolds. Then $f_{t}^{*} \omega_{t}=0$ and $f_{t}^{*} \operatorname{Im}\left(e^{i \theta(t)} \Omega_{t}\right)=0$ for some real valued function $\theta(t):(-\varepsilon, \varepsilon) \rightarrow \mathbb{R}$ with $\theta(0)=0$. At $t=0$,

$$
\begin{aligned}
\frac{d}{d t}\left(f_{t}^{*} \omega_{t}\right) & =\frac{d}{d t}\left(f_{t}^{*}\right) \omega_{t}+f_{t}^{*}\left(\frac{d}{d t} \omega_{t}\right)=f_{0}^{*} L_{W} \omega_{t}+f_{0}^{*} \alpha \\
& =f_{0}^{*} i(W) d \omega_{0}+f_{0}^{*} d i(W) \omega_{0}+f_{0}^{*} \alpha .
\end{aligned}
$$

where $\left.\frac{d}{d t} \omega_{t}\right|_{t=0}=\alpha \in H^{2}\left(X_{0}, \mathbb{C}\right),\left.\frac{d}{d t} f_{t}\right|_{t=0}=W$ is a vector field in $T X_{0}$ defined over $f_{0}(M)$ and $L_{W}$ is Lie derivative.

So

$$
\left.\frac{d}{d t}\left(f_{t}^{*} \omega_{t}\right)\right|_{t=0}=d f_{0}^{*} i(W) \omega_{0}+f_{0}^{*} \alpha .
$$

Note that since we only assume that $f_{0}$ is an immersion, in general $W$ could be multivalued at some points, but anyway we can use the trick in section 1 of $[\mathrm{M}]$ to get around it. We pretend to have $f_{0}$ is an embedding and we identify $M$ with $f_{0}(M)$ in the following. Also note that if we fix the complex 
structure $J_{t}=J$, then $\alpha \in H^{1,1}\left(X_{0}\right)$. Similarly at $t=0$,

$$
\begin{aligned}
\frac{d}{d t} f_{t}^{*} \operatorname{Im}\left(e^{i \theta(t)} \Omega_{t}\right)= & \frac{d}{d t}\left(f_{t}^{*}\right) \operatorname{Im}\left(e^{i \theta(t)} \Omega_{t}\right)+f_{t}^{*} \operatorname{Im}\left(\frac{d}{d t}\left(e^{i \theta(t)} \Omega_{t}\right)\right) \\
= & f_{0}^{*} L_{W} \operatorname{Im} \Omega_{0}+f_{0}^{*} \beta+f_{0}^{*} \operatorname{Im}\left(i \theta^{\prime}(0) \Omega_{0}\right) \\
= & f_{0}^{*} i(W) d \operatorname{Im} \Omega_{0}+f_{0}^{*} d i(W) \operatorname{Im} \Omega_{0} \\
& +f_{0}^{*} \beta+f_{0}^{*} \operatorname{Im}\left(i \theta^{\prime}(0) \Omega_{0}\right) .
\end{aligned}
$$

So

$$
\left.\frac{d}{d t} f_{t}^{*} \operatorname{Im}\left(e^{i \theta(t)} \Omega_{t}\right)\right|_{t=0}=d f_{0}^{*} i(W) \operatorname{Im} \Omega_{0}+f_{0}^{*} \beta+\theta^{\prime}(0) f_{0}^{*} \operatorname{Re} \Omega_{0} .
$$

where $\left.\frac{d}{d t} \operatorname{Im} \Omega_{t}\right|_{t=0}=\beta \in H^{n}\left(X_{0}, \mathbb{R}\right)$.

From (2.1) we see that the obstruction to the deformation of special Lagrangian submanifolds is $f_{0}^{*} H^{2}\left(X_{0}, \mathbb{C}\right)$ when the Kähler class deforms. Since $f_{0}^{*} \operatorname{Re} \Omega_{0}$ is the volume form on $M$, we can make $f_{0}^{*} \beta+\theta^{\prime}(0) f_{0}^{*} \operatorname{Re}$ $\Omega_{0}$ be zero class by choosing $\theta^{\prime}(0)$. Hence it follows from (2.2) that the deformation of special Lagrangian submanifolds is unobstructed when the complex structure deforms. Next we show that infinitesimal deformations can be extended to actual deformations when they are unobstructed. Our proof follows the arguments of $[\mathrm{M}]$.

Theorem 2.1. $\quad$ i) Assume that $\left(X_{t}, \omega_{t}, \Omega_{t}\right)$ is a family of Calabi-Yau triples with fixed cohomology class $\left[\omega_{t}\right]$. Then special Lagrangian submanifold $f_{0}: M \rightarrow X_{0}$ can be extended to a family of special Lagrangian submanifolds $f_{t}: M \rightarrow X_{t}$, for $t \in(-\delta, \delta)$, where $\delta$ is a small positive number.

ii) Assume that $\left(X, \omega_{t}, \Omega\right)$ is a family of Calabi-Yau triples and $f_{0}: M \rightarrow$ $X$ is a special Lagrangian submanifold with respect to $\omega_{0}$. Further assume that $f_{0}^{*} H^{2}(X, \mathbb{C})=0$. Then there are a family of special Lagrangian submanifolds $f_{t}: M \rightarrow X$ with respect to $\omega_{t}$, for $t \in(-\delta, \delta)$, where $\delta$ is a small positive number.

Proof. We prove i) and ii) together by implicit function theorem. The quotient bundle $N_{M \mid X}=f_{0}^{*} T X / T M$ can be viewed as a subbundle of $f_{0}^{*} T X$ using metric $\omega_{0}$. Let $*$ be the Hodge star operator defined by metric $\omega_{0}$. There is an identification between $C^{1, \alpha}\left(\Omega^{1}(M)\right)$ and $C^{1, \alpha}\left(M, N_{M \mid X}\right)$ given by $\phi=i(W)\left(\omega_{0}\right)$. The exponential map below is defined by metric $\omega_{0}$. Let $x$ be an arbitrary point in $M$, consider the map

$$
F:(-\varepsilon, \varepsilon) \times C^{1, \alpha}\left(\Omega^{1}(M)\right) \rightarrow C^{0, \alpha}\left(\Omega^{2}(M)\right) \oplus C^{0, \alpha}\left(\Omega^{n}(M)\right)
$$


defined by

$$
F(t, \phi)=\left(\left(\exp _{x} W(x)\right)^{*} \omega_{t},\left(\exp _{x} W(x)\right)^{*} \operatorname{Im} \Omega_{t}\right)
$$

Here we have absorbed the phase factor $e^{i \theta(t)}$ into $\Omega_{t}$, so that $f_{0}^{*} \frac{d}{d t} \operatorname{Im} \Omega_{t}$ are zero cohomology class on $M$ for all small $t$.

Denote $f_{0}^{*} i(W) \omega_{0}$ by $\psi$. By the identity $\left.f_{0}^{*} i(W) \operatorname{Im} \Omega_{0}\right|_{t=0}=* \psi$ (see $[\mathrm{M}], \S 3)$, the following partial derivative follows from the calculation of (2.1) and (2.2),

$$
D F_{\phi}(0,0)(\psi)=(d \psi+d \tilde{\alpha}, d * \psi+d \tilde{\beta})
$$

where $d \widetilde{\alpha}=\left.f_{0}^{*} \frac{d}{d t} \omega_{t}\right|_{t=0}$ and $d \tilde{\beta}=\left.f_{0}^{*} \frac{d}{d t} \operatorname{Im} \Omega_{t}\right|_{t=0}$. Note that map

$$
D F_{\phi}(0,0): C^{1, \alpha}\left(\Omega^{1}(M)\right) \rightarrow C^{0, \alpha}\left(\Omega^{2}(M)\right) \oplus C^{0, \alpha}\left(\Omega^{n}(M)\right)
$$

can be factored through as a map from $C^{1, \alpha}\left(\Omega^{1}(M)\right)$ into

$$
\begin{aligned}
\operatorname{Im} d\left\{C^{1, \alpha}\left(\Omega^{1}(M)\right)\right. & \left.\rightarrow C^{0, \alpha}\left(\Omega^{2}(M)\right)\right\} \oplus \operatorname{Im} d\left\{C^{1, \alpha}\left(\Omega^{n-1}(M)\right)\right. \\
& \left.\rightarrow C^{0, \alpha}\left(\Omega^{n}(M)\right)\right\} .
\end{aligned}
$$

The key thing is that map $F$ can also be factored through as above. This can be seen as follows: first, $\left[\left(\exp _{x} W(x)\right)^{*} \omega_{t}\right]=\left[f_{0}^{*} \omega_{t}\right]=\left[f_{0}^{*} \omega_{0}\right]=0$. Here we have used the assumption in ii); Second, $\left[\left(\exp _{x} W(x)\right)^{*} \operatorname{Im} \Omega_{t}\right]=\left[f_{0}^{*} \operatorname{Im} \Omega_{t}\right]=$ $\left[f_{0}^{*} \operatorname{Im} \Omega_{0}\right]=0$.

By the Hodge decomposition of $\Omega^{1}(M), D F_{\phi}(0,0)$ is a surjective map, we can apply implicit function theorem to $F$ which maps $(-\varepsilon, \varepsilon) \times C^{1, \alpha}\left(\Omega^{1}(M)\right)$ into

$$
\begin{aligned}
\operatorname{Im} d\left\{C^{1, \alpha}\left(\Omega^{1}(M)\right)\right. & \left.\rightarrow C^{0, \alpha}\left(\Omega^{2}(M)\right)\right\} \oplus \operatorname{Im} d\left\{C^{1, \alpha}\left(\Omega^{n-1}(M)\right)\right. \\
& \left.\rightarrow C^{0, \alpha}\left(\Omega^{n}(M)\right)\right\}
\end{aligned}
$$

So for small $t, F(t, \phi)=0$ has solutions $\phi(t)$. Then the special Lagrangian submanifolds are given by $f_{t}(x)=\exp _{x} W(t, x)$ where $\phi(t)=i(W(t, x))\left(\omega_{0}\right)$.

Remark 2.1. If mirror symmetry holds for Calabi-Yau threefold $X$ with $h^{2,0}(X)=0$, then it switches the moduli of complex structures to the moduli of Kähler structures. We suspect that for any Calabi-Yau threefold $X$ with $h^{2,0}(X)=0, f^{*} H^{2}(X, \mathbb{C})=0$ for special Lagrangian torus $f$ which gives the fibration of $X$, i.e. the deformation of the special Lagrangian torus is unobstructed when Kähler class deforms. 
Example 2.2. $K 3$ surfaces. Here we adopt the notation of [GW], $\S 1$. Things are complicated by $h^{2,0} \neq 0$. Consider the mirror family $S_{M}$, the deformation of Kähler classes satisfies $\frac{d}{d t} \omega_{t} \in M$, but the homology class $\left[f\left(T^{2}\right)\right]$ of the special Lagrangian torus is $E$. So the deformation of the special Lagrangian torus is unobstructed by Theorem 2.1 since E.M $=0$. For the deformation of complex structures within the mirror family $S_{M}$, the family of holomorphic $(2,0)$-forms are

$$
\Omega_{t}=\check{B}_{t}+\frac{E^{\prime}}{m}+\left(\frac{\check{\omega}_{t} \cdot \check{\omega}_{t}-\check{B}_{t} \cdot \check{B}_{t}}{2}\right) E+\sqrt{-1}\left(\check{\omega}_{t}-\left(\check{\omega}_{t} \cdot E\right) E\right),
$$

where $\check{B}_{t}$ and $\check{\omega}_{t}$ belong to $\check{M}$ and $m=1$. So the deformation of the special Lagrangian torus is unobstructed by Theorem 2.1 since $E \cdot \frac{d}{d t} \Omega_{t}=0$.

Example 2.3. Quintic threefolds. Since $h^{1,1}=1$, the Kähler class can not deform. There is no obstruction to the deformation of special Lagrangian submanifolds when complex structures deform by Theorem 2.1 .

\section{Kummer threefold.}

First we describe this Kummer threefold. Let $\xi$ be a primitive cubic root of 1 . Then $E_{\xi}=\mathbb{C} / \mathbb{Z}[1, \xi]$ is the unique elliptic curve which has an automorphism of degree 3, namely $\varphi: E_{\xi} \rightarrow E_{\xi}$ with $\varphi(z)=\xi \cdot z$. For holomorphic map $\Phi: E_{\xi} \times E_{\xi} \times E_{\xi} \rightarrow E_{\xi} \times E_{\xi} \times E_{\xi}$ with

$$
\Phi\left(z_{1}, z_{2}, z_{3}\right)=\left(\xi \cdot z_{1}, \xi \cdot z_{2}, \xi \cdot z_{3}\right),
$$

we have $\Phi^{3}=\operatorname{Id}$ and $\Phi$ has 27 isolated fixed points. Therefore the quotient variety $Y_{0}=E_{\xi} \times E_{\xi} \times E_{\xi} /\{\Phi\}$ has 27 isolated singular points $P_{i}$ of type $\mathbb{C}^{3} / \mathbb{Z}_{3}$. If we blow up these singular points, we get a smooth Calabi-Yau manifold $Y$. We call $Y$ Kummer threefold. Note that $H^{2,1}(Y)=0$, in particular $Y$ has no deformation of the complex structure. Let us first describe the holomorphic $(3,0)$-form on $Y$.

Let $\pi: Y \rightarrow Y_{0}$ be the resolution map. Then $\hat{\Omega}=d z_{1} \wedge d z_{2} \wedge d z_{3}$ is a holomorphic $(3,0)$-form on $E_{\xi} \times E_{\xi} \times E_{\xi}$. Let $\Omega_{0}$ be the holomorphic $(3,0)$-form on $Y_{0}$ induced from $\hat{\Omega}$. Denote $\pi^{*} \Omega_{0}$ by $\Omega$. Then

Lemma 3.1. $\Omega$ is a holomorphic $(3,0)$-form on $Y$.

Proof. We only need to check that $\pi^{*} \Omega_{0}$ extends across the exceptional divisors and is nonzero everywhere on the exceptional divisors. This is a 
local calculation and is true for any dimension. Let $\mathbb{Z}_{n}$ acts on $\mathbb{C}^{n}$ by

$$
\xi \cdot\left(z_{1}, \cdots, z_{n}\right)=\left(\xi \cdot z_{1}, \cdots, \xi \cdot z_{n}\right)
$$

where $\xi$ is a primitive $\mathrm{n}^{\text {th }}$ root of $1 . X_{0}=\mathbb{C}^{n} / \mathbb{Z}_{n}$ has a resolution with trivial canonical bundle. Let $\pi: X \rightarrow X_{0}$ be this resolution map. Then $X$ is the total space of the canonical line bundle $K_{\mathbb{C} P n-1}$ of $\mathbb{C} P^{n-1}$ and the exceptional fiber is $\mathbb{C} P^{n-1}$.

In terms of local coordinates, we consider a coordinate chart $U_{0}$ in $\mathbb{C} P^{n-1}$ :

$$
\begin{aligned}
l & : U_{0}=\mathbb{C}^{n-1} \subset \mathbb{C} P^{n-1} \\
l\left(y_{1}, \cdots, y_{n-1}\right) & =\left[1, y_{1}, \cdots, y_{n-1}\right] .
\end{aligned}
$$

Then

$$
\begin{gathered}
\pi:\left.K_{\mathbb{C} P^{n-1}}\right|_{U_{0}} \rightarrow \mathbb{C}^{n} / \mathbb{Z}_{n} \\
\pi\left(\left[1, y_{1}, \cdots, y_{n-1}\right], \lambda\right)=\left(\lambda^{\frac{1}{n}}, \lambda^{\frac{1}{n}} y_{1}, \cdots, \lambda^{\frac{1}{n}} y_{n-1}\right) .
\end{gathered}
$$

Holomorphic form $d z_{1} \wedge \cdots d z_{n}$ descends to the non-singular part of $X_{0}$. We need to show that $\pi^{*}\left(d z_{1} \wedge \cdots d z_{n}\right)$ extends to whole $X$. To show this, we check it under above local coordinate system.

$$
\begin{aligned}
\pi^{*}\left(d z_{1} \wedge \cdots d z_{n}\right) & =d \lambda^{\frac{1}{n}} \wedge d\left(\lambda^{\frac{1}{n}} y_{1}\right) \wedge \cdots \wedge d\left(\lambda^{\frac{1}{n}} y_{n-1}\right) \\
& =\frac{1}{n} d \lambda \wedge d y_{1} \wedge \cdots \wedge d y_{n-1} .
\end{aligned}
$$

This simple calculation shows directly that $\pi^{*}\left(d z_{1} \wedge \cdots d z_{n}\right)$ extends to an everywhere non-zero holomorphic $n$-form on $X$.

\subsection{Degeneration of Kähler-Einstein metrics.}

Now we describe the Ricci-flat metric on the blowup $\widehat{\mathbb{C}^{3} / \mathbb{Z}_{3}}$ of $\mathbb{C}^{3} / \mathbb{Z}_{3}$ constructed by Calabi ([Ca] ). This metric is $S O(6)$-invariant and asymptotically locally Euclidean. Because of $S O(6)$-symmetry, the Einstein equation for the metric is reduced to an ordinary differential equation for the Kähler potential. Therefore we look for a Ricci-flat Kähler metric on $\mathbb{C}^{3} \backslash(0,0,0)$ in the form $\sqrt{-1} \partial \bar{\partial} h$, where $h=h(u)$ with $u=\left|z_{1}\right|^{2}+\left|z_{2}\right|^{2}+\left|z_{3}\right|^{2}$. The equation for a Ricci-flat Kähler metric is

$$
(\sqrt{-1} \partial \bar{\partial} h)^{3}=\omega_{0}^{3}
$$

where $\omega_{0}=\sqrt{-1} \partial \bar{\partial} u$ is the standard metric on $\mathbb{C}^{3}$. Since

$$
\sqrt{-1} \partial \bar{\partial} h=h^{\prime}(u) \sqrt{-1} \partial \bar{\partial} u+h^{\prime \prime}(u) \sqrt{-1} \partial u \wedge \bar{\partial} u,
$$


the equation (3.1) reduces to

$$
h^{\prime}(u)^{3}+u h^{\prime}(u)^{2} \cdot h^{\prime \prime}(u)=1 .
$$

Solving this equation, we have

$$
\begin{aligned}
& \frac{d\left(u^{3} h^{\prime}(u)^{3}\right)}{d u}=3 u^{2}, \\
& h^{\prime}(u)=\left(1+\frac{a^{3}}{u^{3}}\right)^{1 / 3},
\end{aligned}
$$

where $a$ is a positive constant. Integrating (3.2), we have (subscript $a$ is used to indicate dependence on $a$ )

$$
\begin{aligned}
h_{a}(u)= & u \cdot\left(1+\frac{a^{3}}{u^{3}}\right)^{1 / 3}+\frac{a}{2} \log \left(\left(1+\frac{a^{3}}{u^{3}}\right)^{1 / 3}-\frac{a}{u}\right) \\
& -\frac{a}{\sqrt{3}} \arctan \left(\frac{2 u \cdot\left(1+\frac{a^{3}}{u^{3}}\right)^{1 / 3}+a}{\sqrt{3} a}\right)+c .
\end{aligned}
$$

The function $h_{a}(u)$ behaves asymptotically like $u$ as $u \rightarrow \infty$ and like $a \log u$ as $u \rightarrow 0$. Changing the coordinate under blowup as in Lemma 3.1, by a simple calculation one can see that the Kähler form $\sqrt{-1} \partial \bar{\partial} h_{a}$ extends smoothly across the exceptional divisor of $\widehat{\mathbb{C}^{3} / \mathbb{Z}_{3}}$.

From (3.3) we get the following estimate of metric $\widetilde{g}_{a}$ on $\widehat{\mathbb{C}^{3} / \mathbb{Z}_{3}}$ given by $\sqrt{-1} \partial \bar{\partial} h_{a}$ as $u \rightarrow \infty$ :

$$
\begin{aligned}
h_{a}(u) & =u+O\left(\frac{1}{u}\right), \\
\tilde{g}_{a i \bar{j}} & =\delta_{i j}+O\left(\frac{1}{u^{3}}\right), \\
\partial^{k} \widetilde{g}_{a i \bar{j}} & =O\left(\frac{1}{u^{3+k}}\right) .
\end{aligned}
$$

In particular, the metric is asymptotically locally Euclidean in the sense that

$$
|R m|=O\left(\frac{1}{u^{4}}\right) \text { as } u \rightarrow \infty,
$$

where $R m$ denotes the curvature tensor. To control the curvature for small $u$, we assume that the holomorphic bisectional curvature of metric $\widetilde{g}_{1}$ is 
bounded by $c_{0}$. Note that for any $t \in \mathbb{C}^{*}$ we have scaling property: $\phi_{t}^{*}\left(\frac{\tilde{g}_{a}}{t^{2}}\right)=$ $\tilde{g}_{a / t^{2}}$, where $\phi_{t}: \widehat{\mathbb{C}^{3} / \mathbb{Z}_{3}} \rightarrow \widehat{\mathbb{C}^{3} / \mathbb{Z}_{3}}$ is multiplication by $t$. So as $u \rightarrow 0$, i.e., near exceptional divisor $\mathbb{C} P^{2}$, we have the following estimate of metric $\widetilde{g}_{a}$ :

$$
\text { the holomorphic bisectional curvature of metric } \widetilde{g}_{a} \leq c_{0} / a \text {. }
$$

We now construct the approximated Kähler-Einstein metric on $Y$ by gluing these model metrics to each of the neighborhoods of exceptional divisors of $Y$. For each of singular points $P_{i}$ in $Y_{0}, i=1, \cdots, 27$, we choose a small neighborhood $U_{0 i}$ which is isomorphic to $B_{\delta} / \mathbb{Z}_{3}$ for some fixed positive $\delta \ll 1$, where $B_{\delta}$ denotes a metric ball in $\mathbb{C}^{3}$ of radius $\delta$. Assume $U_{0 i} \cap U_{0 j}=\varnothing$ if $i \neq j$. Let $r=\left(\left|z_{1}\right|^{2}+\left|z_{2}\right|^{2}+\left|z_{3}\right|^{2}\right)^{1 / 2}$ be the distance function on $\mathbb{C}^{3}$ and $t(r)$ be a cut-off function such that: 1) $t(r)=1$ for $r \leq 1-\delta, 2) t(r)=0$ for $r \geq 1$ and 3$)\left|t^{\prime}(r)\right| \leq \frac{2}{\delta}$. If we choose positive numbers $a_{i}$ sufficiently small, then we define Kähler potential $H_{a}(r)$ on $Y$ by

$$
\begin{aligned}
& H_{a}(r)=(1-t(r / \delta)) r^{2}+t(r / \delta) \cdot h_{a_{i}}\left(r^{2}\right) \text { on the resolution } U_{i} \text { of } U_{0 i}, \\
& H_{a}(r)=\left|z_{1}\right|^{2}+\left|z_{2}\right|^{2}+\left|z_{3}\right|^{2} \text { on the complement } Y \backslash\left(\cup_{i} U_{i}\right) .
\end{aligned}
$$

It is easy to see that $\omega_{a}=\sqrt{-1} \partial \bar{\partial} H_{a}$ defines a Kähler metric $g_{a}$ on $Y$ since we are gluing along almost flat part of two metrics on neighborhoods of exceptional divisors. The metric $g_{a}$ is not Ricci-Flat only in the 27 neck regions $N_{i}$ defined by $t(r / \delta) \cdot(1-t(r / \delta)) \neq 0$. Denote $\max _{i}\left\{1 / a_{i}\right\}$ by $c_{a}$. From (3.4) and (3.5) we have the following estimate of metric $g_{a}$ :

$$
\text { the holomorphic bisectional curvature of metric } g_{a} \leq c_{0} c_{a} \text {. }
$$

Yau's existence theorem of Ricci-flat Kähler metrics says that the following Monge-Ampère equation has an unique solution $u_{a}$ up to a constant:

$$
\left(\omega_{a}+\sqrt{-1} \partial \bar{\partial} u_{a}\right)^{3}=e^{f_{a}} \cdot \omega_{a}^{3},
$$

where $f_{a}$ is defined by $f_{a}=\log \left(\frac{\Omega \wedge \bar{\Omega}}{\omega_{a}^{3}}\right)$. From (3.4) a direct computation shows that the function $f_{a}=a_{i}^{3} \cdot \tilde{f}_{a}$ on the neck $N_{i}$ where smooth function $\tilde{f}_{a}$ and their derivative is bounded uniformly on $N_{i}$. From now on $C$ is some constant independent of $a_{i}$ which may vary from place to place. We have

$$
\left\|f_{a}\right\|_{C^{2, \alpha}} \leq C \cdot|a|^{3}
$$

where $|a|^{2}=\sum_{1}^{27}\left|a_{i}\right|^{2}$. 
Before we derive estimates for the solution $u_{a}$ of (3.8). We need the following two lemmas. Sobolev inequality plays a central role in deriving estimates for the solutions of Monge-Ampère equation (3.8). The following Sobolev lemma follows from Theorem 13 in C. Croke's paper [Cr].

Lemma 3.2. Let $(M, g)$ be a compact Riemannian manifold of dimension $m$ without boundary and $V$ be the volume. Then there exist two positive constants $c_{1}$ and $c_{2}$ such that for any $f$ in Sobolev space $H^{1}(M)$,

$$
c_{1} \cdot V^{2 / m}\left(\int_{M}|f|^{\frac{2 m}{m-2}} d V\right)^{\frac{m-2}{m}}-c_{2} \cdot \int_{M}|f|^{2} d V \leq \int_{M}|d f|^{2} d V
$$

where $c_{1}$ and $c_{2}$ depend only on the dimension of $M$, the volume of $M$, the diameter of $M$ and a lower bound on the Ricci curvature of $M$.

The proof of the next lemma belongs to Peter $\mathrm{Li}$ (see also [L]). It is communicated to us by Naichung C. Leung.

Lemma 3.3. Let $(M, g)$ be a compact Riemannian manifold without boundary and $G(x, y)$ be the Green function of Laplacian. Assume that

$$
\int_{M} G(x, y) d V(x)=0 .
$$

Then there exists a constant $c$ such that

$$
G(x, y) \geq-c,
$$

where $c$ depends only on the dimension of $M$, the volume of $M$, the diameter of $M$ and a lower bound on the Ricci curvature of $M$.

Proof. The Green's function $G(x, y)$ can be obtained by integrating

$$
H(x, y, t)-\frac{1}{V}
$$

over the variable $0<t<\infty$, where $H(x, y, t)$ is the heat kernel and $V$ is the volume of $M$. Let

$$
K(x, y, t)=H(x, y, t)-\frac{1}{V} .
$$

Clearly

$$
\int_{0}^{1} K(x, y, t) d t \geq-\frac{1}{V}
$$


Hence we only need to estimate the integral over $1<t<\infty$.

The semi-group property says that

$$
K(x, y, 2 t)=\int_{M} K(x, z, t) K(z, y, t) d z .
$$

Applying Schwartz inequality on the right hand side, we have

$$
\begin{aligned}
|K(x, y, 2 t)| \leq & \left(\int_{M} K^{2}(x, z, t) d z\right)^{1 / 2} \cdot\left(\int_{M} K^{2}(z, y, t) d z\right)^{1 / 2} \\
= & \left(\int_{M} K(x, z, t) K(z, x, t) d z\right)^{1 / 2} \cdot \\
& \cdot\left(\int_{M} K(z, y, t) K(y, z, t) d z\right)^{1 / 2} \\
= & K(x, x, 2 t)^{1 / 2} \cdot K(y, y, 2 t)^{1 / 2} \\
K(x, y, t) \geq & -K^{1 / 2}(x, x, t) K^{1 / 2}(y, y, t) .
\end{aligned}
$$

On the other hand, to estimate $K(x, x, t)$ we differentiate the semi-group property with respect to $t$ and get

$$
\begin{aligned}
K^{\prime}(x, x, 2 t) & =2 \int_{M} K(x, z, t) \cdot K^{\prime}(z, x, t) d z=2 \int_{M} K(x, z, t) \cdot \Delta K(z, x, t) d z \\
& =-2 \int_{M}|\nabla K(x, z, t)|^{2} d z .
\end{aligned}
$$

Since $\int_{M} K(x, z, t) d z=0$, the Poincare inequality implies that

$$
\int_{M}|\nabla K(x, z, t)|^{2} d z \geq \lambda \int_{M} K^{2}(x, z, t) d z=\lambda K(x, x, 2 t),
$$

where $\lambda$ is the first eigenvalue. This implies that

$$
K^{\prime}(x, x, t) \leq-2 \lambda K(x, x, t),
$$

hence

$$
K(x, x, t) \leq K(x, x, 1) e^{-2 \lambda(t-1)}
$$

for all $t \geq 1$. However,

$$
K(x, x, 1)=H(x, x, 1)-\frac{1}{V} \leq H(x, x, 1) .
$$


Now the upper bound of $H(x, y, 1)$ and the lower bound of $\lambda$ give an estimate of $K(x, y, t)$ which is integrable over $1 \leq t<\infty$. Note that both the upper bound of $H(x, x, 1)$ and the lower bound of $\lambda$ only depend on the lower bound of Ricci curvature and the diameter (see, for example, [SY] Theorem 4.6, p169 and Theorem 4, p116 respectively). The lemma is proved.

Since the family of metrics $g_{a}$ has uniformly bound on the volume, the diameter and a lower bound on the Ricci curvature, the positive constants $c_{1}$ and $c_{2}$ in (3.10) and $c$ in (3.11) can be chosen independent of $a_{i}$ for the family of metrics $g_{a}$.

We begin with the $C^{0}$-estimate of $u_{a}$. If we only need the uniform bound of $u_{a}$, we can do it in the way of [T], p157 (see also [Y]). However we need $\left|u_{a}\right|$ to be small which is necessary in the second derivative estimate. We modify their proof. Without loss of generality, we may assume that $\int_{Y} u_{a} \omega_{a}^{3}=0$. Denote $\omega_{a}+\sqrt{-1} \partial \bar{\partial} u_{a}$ by $\widetilde{\omega}_{a}$. Let $V_{a}=\int_{Y} \omega_{a}^{3}$. Then

$$
\begin{aligned}
\frac{1}{V_{a}} \int_{Y} u_{a}\left(\widetilde{\omega}_{a}^{3}-\omega_{a}^{3}\right) & =\frac{1}{V_{a}} \int_{Y} u_{a} \sqrt{-1} \partial \bar{\partial} u_{a} \wedge\left(\widetilde{\omega}_{a}^{2}+\widetilde{\omega}_{a} \wedge \omega_{a}+\omega_{a}^{2}\right) \\
& =-\frac{1}{V_{a}} \int_{Y} \sqrt{-1} \partial u_{a} \wedge \bar{\partial} u_{a} \wedge\left(\widetilde{\omega}_{a}^{2}+\widetilde{\omega}_{a} \wedge \omega_{a}+\omega_{a}^{2}\right) \\
& \leq-\frac{1}{V_{a}} \int_{Y} \sqrt{-1} \partial u_{a} \wedge \bar{\partial} u_{a} \wedge \omega_{a}^{2} \\
& =-\frac{1}{V_{a}} \int_{Y}\left|\nabla u_{a}\right|^{2} \omega_{a}^{3} .
\end{aligned}
$$

Note that

$$
\frac{1}{V_{a}} \int_{Y} u_{a}\left(\widetilde{\omega}_{a}^{3}-\omega_{a}^{3}\right)=\frac{1}{V_{a}} \int_{Y} u_{a}\left(e^{f_{a}}-1\right) \omega_{a}^{3} .
$$

By applying (3.9) and Hölder inequality, we have

$$
\begin{aligned}
\frac{1}{V_{a}} \int_{Y}\left|\nabla u_{a}\right|^{2} \omega_{a}^{3} & \leq \frac{1}{V_{a}} \int_{Y}\left|u_{a}\left(e^{f_{a}}-1\right)\right| \omega_{a}^{3} \\
& \leq \frac{C \cdot|a|^{3}}{V_{a}} \int_{Y}\left|u_{a}\right| \omega_{a}^{3} \leq C \cdot|a|^{3}\left(\frac{1}{V_{a}} \int_{Y}\left|u_{a}\right|^{2} \omega_{a}^{3}\right)^{1 / 2} .
\end{aligned}
$$

By Poincare inequality (here we use the first eigenvalue has an uniform bound for metric $g_{a}$ ), we deduce

$$
\frac{(\lambda)^{2}}{V_{a}} \int_{Y}\left|u_{a}\right|^{2} \omega_{a}^{3} \leq C \cdot|a|^{3}\left(\frac{1}{V_{a}} \int_{Y}\left|u_{a}\right|^{2} \omega_{a}^{3}\right)^{1 / 2} .
$$


Let

We have proved

$$
\left\|u_{a}\right\|_{p}=\left(\frac{1}{V_{a}} \int_{Y}\left|u_{a}\right|^{p} \omega_{a}^{3}\right)^{1 / p}
$$

$$
\left\|u_{a}\right\|_{2} \leq C \cdot|a|^{3}
$$

Denote $u_{a}^{+}=\max \left(u_{a}, 0\right)$ and $u_{a}^{-}=\max \left(-u_{a}, 0\right)$. A direct computation gives

$$
\begin{aligned}
& \frac{1}{V_{a}} \int_{Y}\left(u_{a}^{-}\right)^{p}\left(\omega_{a}^{3}-\widetilde{\omega}_{a}^{3}\right) \\
&=-\frac{1}{V_{a}} \int_{Y}\left(u_{a}^{-}\right)^{p} \sqrt{-1} \partial \bar{\partial} u_{a} \wedge\left(\widetilde{\omega}_{a}^{2}+\widetilde{\omega}_{a} \wedge \omega_{a}+\omega_{a}^{2}\right) \\
&=-\frac{4 p}{(p+1)^{2}} \cdot \\
& \quad \cdot \frac{1}{V_{a}} \int_{Y} \sqrt{-1} \partial\left(\left(u_{a}^{-}\right)^{\frac{p+1}{2}}\right) \wedge \bar{\partial}\left(\left(u_{a}^{-}\right)^{\frac{p+1}{2}}\right) \wedge\left(\widetilde{\omega}_{a}^{2}+\widetilde{\omega}_{a} \wedge \omega_{a}+\omega_{a}^{2}\right) \\
& \leq-\frac{4 p}{(p+1)^{2}} \cdot \frac{1}{V_{a}} \int_{Y} \sqrt{-1} \partial\left(\left(u_{a}^{-}\right)^{\frac{p+1}{2}}\right) \wedge \bar{\partial}\left(\left(u_{a}^{-}\right)^{\frac{p+1}{2}}\right) \wedge \omega_{a}^{2} \\
&=-\frac{4 p}{(p+1)^{2}} \cdot \frac{1}{V_{a}} \int_{Y}\left|\nabla\left(\left(u_{a}^{-}\right)^{\frac{p+1}{2}}\right)\right|^{2} \cdot \omega_{a}^{3},
\end{aligned}
$$

and

$$
\begin{aligned}
& \frac{1}{V_{a}} \int_{Y}\left(u_{a}^{+}\right)^{p}\left(\widetilde{\omega}_{a}^{3}-\omega_{a}^{3}\right) \\
&=\frac{1}{V_{a}} \int_{Y}\left(u_{a}^{+}\right)^{p} \sqrt{-1} \partial \bar{\partial} u_{a} \wedge\left(\widetilde{\omega}_{a}^{2}+\widetilde{\omega}_{a} \wedge \omega_{a}+\omega_{a}^{2}\right) \\
&=-\frac{4 p}{(p+1)^{2}} \cdot \\
& \cdot \frac{1}{V_{a}} \int_{Y} \sqrt{-1} \partial\left(\left(u_{a}^{+}\right)^{\frac{p+1}{2}}\right) \wedge \bar{\partial}\left(\left(u_{a}^{+}\right)^{\frac{p+1}{2}}\right) \wedge\left(\widetilde{\omega}_{a}^{2}+\widetilde{\omega}_{a} \wedge \omega_{a}+\omega_{a}^{2}\right) \\
& \leq-\frac{4 p}{(p+1)^{2}} \cdot \frac{1}{V_{a}} \int_{Y} \sqrt{-1} \partial\left(\left(u_{a}^{+}\right)^{\frac{p+1}{2}}\right) \wedge \bar{\partial}\left(\left(u_{a}^{+}\right)^{\frac{p+1}{2}}\right) \wedge \omega_{a}^{2} \\
&=-\frac{4 p}{(p+1)^{2}} \cdot \frac{1}{V_{a}} \int_{Y}\left|\nabla\left(\left(u_{a}^{+}\right)^{\frac{p+1}{2}}\right)\right|^{2} \cdot \omega_{a}^{3} .
\end{aligned}
$$

Combining these two inequalities, we have

$$
\begin{aligned}
\frac{4 p}{(p+1)^{2}} \cdot \frac{1}{V_{a}} \int_{Y}\left|\nabla\left(\left(\left|u_{a}\right|\right)^{\frac{p+1}{2}}\right)\right|^{2} \cdot \omega_{a}^{3} & \leq \frac{C}{V_{a}} \int_{Y}\left|u_{a}\right|^{p} \cdot\left|e^{f_{a}}-1\right| \cdot \omega_{a}^{3} \\
& \leq C \cdot|a|^{3} \cdot \frac{1}{V_{a}} \int_{Y}\left|u_{a}\right|^{p} \cdot \omega_{a}^{3} .
\end{aligned}
$$


It follows from this and Sobolev inequality (3.10) that

$$
\begin{aligned}
\frac{4 p}{(p+1)^{2}}\left\{c_{1}\left(\frac{1}{V_{a}} \int_{Y}\left|u_{a}\right|^{\frac{3(p+1)}{2}} \omega_{a}^{3}\right)^{2 / 3}\right. & \left.-\frac{c_{2}}{V_{a}} \int_{Y}\left|u_{a}\right|^{p+1} \omega_{a}^{3}\right\} \\
& \leq C \cdot|a|^{3} \cdot \frac{1}{V_{a}} \int_{Y}\left|u_{a}\right|^{p} \omega_{a}^{3}
\end{aligned}
$$

Let $p_{0}=2$ and $p_{i}=\frac{3}{2} p_{i-1}$, for $i \geq 1$. It follows from (3.13) that

$$
\left\|u_{a}\right\|_{p_{i}}^{p_{i-1}} \leq \frac{c_{2}}{c_{1}}\left\|u_{a}\right\|_{p_{i-1}}^{p_{i-1}}+\frac{C \cdot p_{i-1}^{2}}{4 c_{1} \cdot\left(p_{i-1}-1\right)} \cdot \frac{1}{V_{a}} \int_{Y}\left|u_{a}\right|^{p_{i-1}-1} \omega_{a}^{3} \cdot|a|^{3} .
$$

Assume that $\left\|u_{a}\right\|_{p_{i-1}} \leq C_{i-1} \cdot|a|^{3}$ (we assume $C_{i-1} \geq 1$ ). Then by Hölder inequality

$$
\begin{aligned}
\left\|u_{a}\right\|_{p_{i}}^{p_{i-1} \leq} \leq & \frac{c_{2}}{c_{1}} C_{i-1}^{p_{i-1} \cdot|a|^{3 p_{i-1}}} \\
& +\frac{C \cdot p_{i-1}^{2}}{4 c_{1} \cdot\left(p_{i-1}-1\right)}\left(\frac{1}{V_{a}} \int_{Y}\left|u_{a}\right|^{p_{i-1}} \omega_{a}^{3}\right)^{\left(p_{i-1}-1\right) / p_{i-1}} \cdot|a|^{3} \\
\leq & \left(\frac{c_{2}}{c_{1}} C_{i-1}^{p_{i-1}}+\frac{C \cdot p_{i-1}^{2}}{4 c_{1} \cdot\left(p_{i-1}-1\right)} C_{i-1}^{p_{i-1}-1}\right)|a|^{3 p_{i-1}}
\end{aligned}
$$

Note that

$$
\frac{c_{2}}{c_{1}} C_{i-1}^{p_{i-1}}+\frac{C \cdot p_{i-1}^{2}}{4 c_{1} \cdot\left(p_{i-1}-1\right)} C_{i-1}^{p_{i-1}-1} \leq C \cdot p_{i-1} C_{i-1}^{p_{i-1}} .
$$

We can choose $C_{i}=\left(C \cdot p_{i-1}\right)^{1 / p_{i-1}} \cdot C_{i-1}$. Using (3.12), it follows that $C_{i}$ has a uniform bound $C$. We have proved

$$
\left\|u_{a}\right\|_{p_{i}} \leq C \cdot|a|^{3} .
$$

Now the $C^{0}$-estimate follows by letting $i \rightarrow \infty$,

$$
\left\|u_{a}\right\|_{C^{0}} \leq C \cdot|a|^{3}
$$

where $C$ is independent of $a$.

Now we derive the $C^{2}$-estimate for $u_{a}$. Let $\Delta_{a}$ and $\widetilde{\Delta}_{a}$ be Laplacians given by metrics $\omega_{a}$ and $\widetilde{\omega}_{a}$ respectively. By (3.7) $c_{0} \cdot c_{a}$ bounds the holomorphic bisectional curvature of metric $\omega_{a}$. Let

$$
k(x)=-\inf _{i \neq j}\left(-R_{i \bar{i} j \bar{j}}\right)(x) / c_{a} .
$$


Then $|k(x)| \leq c_{0}$. The following inequality is $(2.22)$ in $[\mathrm{Y}]$.

$$
\begin{aligned}
e^{2 \widetilde{c}_{a} u_{a}} \widetilde{\Delta}_{a}\left(e^{-2 \widetilde{c}_{a} u_{a}}\right. & \left.\left(3+\Delta_{a} u_{a}\right)\right)(x) \\
& \geq A(x)+B(x)\left(3+\Delta_{a} u_{a}\right)+C(x)\left(3+\Delta_{a} u_{a}\right)^{3 / 2}
\end{aligned}
$$

where

$$
\begin{aligned}
A(x) & =\Delta_{a} f_{a}(x)-9 \inf _{i \neq j}\left(-R_{i \bar{i} \bar{j} \bar{j}}\right)(x), \\
B(x) & =-6 \widetilde{c}_{a}, \\
C(x) & =\left(2 \widetilde{c}_{a}+\inf _{i \neq j}\left(-R_{i \bar{i} \bar{j} \bar{j}}\right)(x)\right) e^{-f_{a}(x) / 2}, \\
\widetilde{c}_{a} & =\frac{1}{2}\left(1+c_{0}\right) c_{a} .
\end{aligned}
$$

We try to get an estimate of $3+\Delta_{a} u_{a}$ from (3.16). Suppose that $e^{-2 \widetilde{c}_{a} u_{a}}(3+$ $\Delta_{a} u_{a}$ ) attains its maximum at $x \in Y$. Then from (3.16)

$$
\begin{aligned}
0 \geq & e^{2 \widetilde{c}_{a} u_{a}} \widetilde{\Delta}_{a}\left(e^{-2 \widetilde{c}_{a} u_{a}}\left(3+\Delta_{a} u_{a}\right)\right)(x) \\
\geq & \Delta_{a} f_{a}(x)+9 k(x) c_{a}-6 \widetilde{c}_{a}\left(3+\Delta_{a} u_{a}\right)(x) \\
& +e^{-f_{a}(x) / 2}\left(1+c_{0}-k(x)\right) c_{a}\left(3+\Delta_{a} u_{a}\right)^{3 / 2}(x) .
\end{aligned}
$$

Hence

$$
\begin{aligned}
& \text { either } e^{-f_{a}(x) / 2}\left(1+c_{0}-k(x)\right) c_{a}\left(3+\Delta_{a} u_{a}\right)^{3 / 2}(x) \\
& \leq 12 \widetilde{c}_{a}\left(3+\Delta_{a} u_{a}\right)(x) \\
& \text { or } e^{-f_{a}(x) / 2}\left(1+c_{0}-k(x)\right) c_{a}\left(3+\Delta_{a} u_{a}\right)^{3 / 2}(x) \\
& \leq-2\left(\Delta_{a} f_{a}(x)+9 k(x) c_{a}\right) .
\end{aligned}
$$

By (3.9) it implies that

$$
\left(3+\Delta_{a} u_{a}\right)(x) \leq C .
$$

So we have proved for any $y \in Y$ using (3.15)

$$
\left(3+\Delta_{a} u_{a}\right)(y) \leq e^{2 \widetilde{c}_{a}\left(\sup u_{a}-\inf u_{a}\right)}\left(3+\Delta_{a} u_{a}\right)(x) \leq C,
$$

when $\max \left\{a_{i}\right\} / \min \left\{a_{i}\right\}$ is bound by an arbitrary fixed number.

The equation (3.8) and estimate (3.9) imply

$$
3+\Delta_{a} u_{a}=\sum_{i}\left(1+u_{i \bar{i}}\right) \geq 3\left(\prod_{i}\left(1+u_{i \bar{i}}\right)\right)^{1 / 3}=3 e^{f_{a}(x) / 3} \geq 3\left(1-C \cdot|a|^{2}\right) .
$$


From this, (3.17), (3.9) and equation (3.8) it follows

$$
C^{-1} \cdot \omega_{a} \leq \widetilde{\omega}_{a} \leq C \cdot \omega_{a} .
$$

This is the $C^{2}$-estimate for $u_{a}$.

It is well-known that $C^{2, \alpha}$-estimate follows directly from (3.18):

$$
\left\|u_{a}\right\|_{C^{2, \alpha}} \leq C \text {. }
$$

(see Theorem 17.14 in [GT] and it is easy to check that the constant $C$ is independent of $a$ 's.)

We need to improve estimate (3.19). We follow the approach of $R$. Kobayashi $([\mathrm{K}])$. Consider the following equation with parameter $t$ :

$$
\begin{aligned}
\left(\omega_{a}+\sqrt{-1} \partial \bar{\partial} u_{a, t}\right)^{3} & =\left(1+t\left(e^{f_{a}}-1\right)\right) \cdot \omega_{a}^{3}, \\
\int_{Y} u_{a, t} \cdot \omega_{a}^{3} & =0 .
\end{aligned}
$$

It has an unique solution by $[Y]$. Since $\log \left(1+t\left(e^{f_{a}}-1\right)\right)$ has the same properties as $f_{a}$, the above discussion implies that there is $C^{2, \alpha}$ estimate like (3.19) for $u_{a, t}$ :

$$
\left\|u_{a, t}\right\|_{C^{2, \alpha}} \leq C,
$$

where constant $C$ is independent of $t \in[0,1]$ and $a$. Differentiating the equation (3.20) with respect to $t$, we have

$$
\Delta_{a, t} \frac{d u_{a, t}}{d t}=\frac{e^{f_{a}}-1}{1+t\left(e^{f_{a}}-1\right)} .
$$

Let $\widetilde{G}_{a, t}(x, y)$ be the Green's function for the Laplacian of metric $\omega_{a, t}=$ $\omega_{a}+\sqrt{-1} \partial \bar{\partial} u_{a, t}$ with $\inf _{Y} \widetilde{G}_{a, t}(x, y)=1$. Then by Green's formula,

$$
\frac{d u_{a, t}(x)}{d t}=\frac{1}{V_{a, t}} \int_{Y} \frac{d u_{a, t}(y)}{d t} \omega_{a, t}^{3}-\frac{1}{V_{a, t}} \int_{Y} \Delta_{a, t} \frac{d u_{a, t}(y)}{d t} \cdot \widetilde{G}_{a, t}(x, y) \omega_{a, t}^{3}
$$

where $V_{a, t}=\int_{Y} \omega_{a, t}^{3}$. So

$$
\begin{aligned}
\left|\frac{d u_{a, t}(x)}{d t}\right| \leq & \left|\frac{1}{V_{a, t}} \int_{Y} \frac{d u_{a, t}(y)}{d t} \omega_{a}^{3}\right|+\left|\frac{1}{V_{a, t}} \int_{Y} \frac{d u_{a, t}(y)}{d t}\left(\omega_{a, t}^{3}-\omega_{a}^{3}\right)\right| \\
& +\left|\frac{1}{V_{a, t}} \int_{Y} \Delta_{a, t} \frac{d u_{a, t}(y)}{d t} \cdot \widetilde{G}_{a, t}(x, y) \omega_{a, t}^{3}\right| .
\end{aligned}
$$


Now we estimate each term on the right side. The first integral is 0 by the second equation in (3.20). As for the second integral,

$$
\begin{aligned}
\left|\frac{1}{V_{a, t}} \int_{Y} \frac{d u_{a, t}(y)}{d t}\left(\omega_{a, t}^{3}-\omega_{a}^{3}\right)\right| & \leq\left|\frac{d u_{a, t}(x)}{d t}\right|_{C^{0}} \cdot \frac{1}{V_{a, t}} \int_{Y} t\left|e^{f_{a}}-1\right| \omega_{a}^{3} \\
& \leq C|a|^{3} \cdot\left|\frac{d u_{a, t}(x)}{d t}\right|_{C^{0}} \\
& \leq \frac{1}{2}\left|\frac{d u_{a, t}(x)}{d t}\right|_{C^{0}} .
\end{aligned}
$$

when $a$ is small. Because of (3.21), Lemma 3.3 implies that there is a constant $C$ independent of $a$ and $t$ such that

$$
\frac{1}{V_{a, t}} \int_{Y} \widetilde{G}_{a, t}(x, y) \omega_{a, t}^{3} \leq C .
$$

This and

$$
\left|\frac{e^{f_{a}}-1}{1+t\left(e^{f_{a}}-1\right)}\right| \leq C \cdot|a|^{3}
$$

imply the third integral is bounded by $C \cdot|a|^{3}$. So we get

$$
\begin{aligned}
&\left|\frac{d u_{a, t}}{d t}\right|_{C^{0}} \leq \frac{1}{2}\left|\frac{d u_{a, t}}{d t}\right|_{C^{0}}+C \cdot|a|^{3}, \\
&\left|\frac{d u_{a, t}}{d t}\right|_{C^{0}} \leq C \cdot|a|^{3} .
\end{aligned}
$$

Denote the Hölder norm with respect to metric $\omega_{a}$ by $C^{k, \alpha}$ and Hölder norm with respect to a fixed coordinate system on $Y$ by $\widetilde{C}^{k, \alpha}$. Note that near the exceptional divisor metric $g_{a}$ goes to zero at a rate $|a|$. Now it follows from the interior Schauder estimate for equation (3.22), (3.9) and (3.24) that

$$
\begin{aligned}
\left\|\frac{d u_{a, t}}{d t}\right\|_{C^{2, \alpha}} & \leq C \cdot|a|^{3}, \\
\left\|\frac{d u_{a, t}}{d t}\right\|_{\widetilde{C}^{2}} & \leq C \cdot|a|^{2}, \\
\left\|\frac{d u_{a, t}}{d t}\right\|_{\widetilde{C}^{2, \alpha}(K)} & \leq C \cdot|a|^{3},
\end{aligned}
$$

where $K$ is any relatively compact set in the complement of the 27 exceptional divisor $\mathbb{C} P^{2}$ 's, $C$ is a constant independent of $t$ and $a_{i}$ but depending 
on $K$ and $\max \left\{a_{i}\right\} / \min \left\{a_{i}\right\}$. Note that $u_{a, 0}=0$ and $u_{a, 1}=u_{a}$. From $u_{a}=\int_{0}^{1} \frac{d u_{a, t}}{d t} d t$ and (3.25), we have

$$
\begin{aligned}
\left\|u_{a}\right\|_{C^{2, \alpha}} & \leq C \cdot|a|^{3}, \\
\left\|u_{a}\right\|_{\widetilde{C}^{2}} & \leq C \cdot|a|^{2} \\
\left\|u_{a}\right\|_{\widetilde{C}^{2, \alpha}(K)} & \leq C \cdot|a|^{3} .
\end{aligned}
$$

It follows by bootstrapping argument that

$$
\begin{aligned}
\left\|u_{a}\right\|_{C^{4, \alpha}} & \leq C \cdot|a|^{3}, \\
\left\|u_{a}\right\|_{\widetilde{C}^{3}} & \leq C \cdot|a|^{3 / 2}, \\
\left\|u_{a}\right\|_{\widetilde{C}^{4}} & \leq C \cdot|a|, \\
\left\|u_{a}\right\|_{\widetilde{C}^{4, \alpha}(K)} & \leq C \cdot|a|^{3} .
\end{aligned}
$$

We have proved the following

Theorem 3.4. Let $Y_{0}$ and $Y$ be the space defined at the beginning of this section. Let $\omega_{a}$ be the Kähler metric defined by (3.6). Then, for any relatively compact set $K$ in the complement of the 27 exceptional divisor $\mathbb{C} P^{2}$ 's, there exist positive constants $C_{1}$ and $C_{2}$ both independent of a (but $C_{1}$ depending on $K$ and $\max \left\{a_{i}\right\} / \min \left\{a_{i}\right\}$ and $C_{2}$ depending on $\left.\max \left\{a_{i}\right\} / \min \left\{a_{i}\right\}\right)$ such that

$$
\begin{aligned}
\left\|u_{a}\right\|_{\widetilde{C}^{4, \alpha}(K)} & \leq C_{1} \cdot|a|^{3}, \\
\left\|u_{a}\right\|_{C^{4, \alpha}(K)} & \leq C_{2} \cdot|a|^{3} .
\end{aligned}
$$

\subsection{Special Lagrangian tori in $Y$.}

In this subsection we prove the existence of a family of special Lagrangian tori in $Y$. First $E_{\xi} \times E_{\xi} \times E_{\xi}$ has special Lagrangian torus fibration with respect to holomorphic $(3,0)$-form $\hat{\Omega}=d z_{1} \wedge d z_{2} \wedge d z_{3}$ and flat metric, namely $T_{a, b, c}=T_{a} \times T_{b} \times T_{c}$ for any real numbers $a, b$ and $c$. Here $T_{a} \subset E_{\xi}$ is the image of $\mathbb{R}+a \sqrt{-1}$ under the projection $\mathbb{C} \rightarrow E_{\xi}$. For generic values of $a, b$ and $c$, the image of $T_{a, b, c}$ in $Y_{0}$ does not meet those $P_{i}$ 's and gives an immersed torus. It is in fact a special Lagrangian torus with respect to the quotient metric, which is a degenerate Kähler-Einstein metric, and the holomorphic form $\Omega_{0}$. Now we conclude that this immersed torus in $Y_{0}$ can be perturbed to a special Lagrangian torus in $Y$. In fact the same is true for any special Lagrangian submanifold in $Y_{0} \backslash\left\{P_{i}\right\}$. 
Theorem 3.5. Let $Y$ be Kummer threefold. Then any special Lagrangian torus $f_{0}$ in $Y_{0} \backslash\left\{P_{i}\right\}$ can be perturbed to a special Lagrangian torus in $Y$.

Proof. Assume that open set $U$ contains the image $f_{0}$ in $Y_{0} \backslash\left\{P_{i}\right\}$ and the closure $\bar{U}$ of $U$ is compact. We have shown that $\pi^{*} \Omega_{0}$ is a holomorphic $(3,0)$-form on $Y$.

Now the Ricci-flat metric $\widetilde{\omega}_{a}=\omega_{a}+\sqrt{-1} \partial \bar{\partial} u_{a}$ differs from $\omega_{a}$ by a exact form $\sqrt{-1} \partial \bar{\partial} u_{a}$ on $\bar{U}$ which is small by Theorem 3.4 and $\pi^{*} \Omega_{0}$ is the same as $\Omega_{0}$ on $\bar{U}$. From the proof of Theorem 2.1, we conclude that we can deform special Lagrangian torus $f_{0}$ to a special Lagrangian torus in $\left(Y, \widetilde{\omega}_{a}, \pi^{*} \Omega_{0}\right)$ when we choose $a_{i}$ small enough and $\max \left\{a_{i}\right\} / \min \left\{a_{i}\right\}$ bounded.

Remark 3.2. Note that Kummer surfaces are constructed exactly the same way as $Y$. Theorem 3.4 is true for Kummer surfaces (see $[\mathrm{K}]$, Theorem 18) and the relation between holomorphic (3,0)-form is also true for Kummer surfaces (holomorphic $(2,0)$-form then). So we get the existence of a family of special Lagrangian tori on Kummer surfaces without using elliptic fibration method of [HL].

\section{References.}

[B] R.L. Bryant, Submanifolds and special structures on the octonians, J. Diff. Geom. 17 (1982), 185-232.

[Ca] E. Calabi, Metriques Kählériennes et fibré holomorphes, Ann. Sci. Éc. Norm. Sup. 4, 12 (1979), 269-294.

[Cr] C. Croke, Some isoperimetric inequalities and eigenvalue estimates, Ann. Sci. Éc. Norm. Sup. 4, 13 (1980), 419-435.

[GT] D. Gilbarg and N.S. Trudinger, Elliptic partial differential equations of second order, Springer-Verlag, New York 1983.

[GW] M. Gross and P.M.H. Wilson, Mirror symmetry via 3-tori for a class of Calabi-Yau threefolds, to appear in Math. Ann. alg-geom/9608004.

[HL] R. Harvey and H.B. Lawson, Jr., Calibrated geometries Acta math. 148 (1982), 47-157.

[K] R. Kobayashi, Moduli of Einstein metrics on a K3 surface and degeneration of type I, in Adv. Studies in Pure Math. 18-II (1990), 257-311. 
[L] N.C. Lee, Estimate for heat kernel and Green's function, in Tsing Hua Lectures on Geometry and Analysis, ed. by S.T. Yau, International Press, Cambridge, MA 1997.

[M] R.C. McLean, Deformation of calibrated submanifolds, to appear in J. Diff. Geom.

[P] D. Page, A physical picture of the K3 gravitational instanton, Phys. Lett. $80 \mathrm{~B}$ (1978), 55-57.

[R] S.S. Roan, On the generalization of Kummer surfaces, J. Diff. Geom. 30 (1989), 523-537.

[SY] R. Schoen and S.T. Yau, Lectures on Differential Geometry, International Press, Cambridge, MA 1994.

[SYZ] A. Strominger, S.T. Yau and E. Zaslow, Mirror symmetry is T-duality, Nucl. Phys. B479 (1996), 243-259.

[T] G. Tian, Kähler-Einstein metrics on algebraic manifolds, in Transcendental Methods in Algebraic Geometry, ed. by F. Catanese and C. Ciliberto, Lectures Notes in Math. 1646, Springer, New York 1996, 143-185.

[Y] S.T. Yau, On the Ricci curvature of a compact Kähler manifold and the complex Monge-Ampère equations, I, Comm. Pure Appl. Math. 31 (1978), $339-411$.

RECEIVED November 20, 1997.

UNIVERSITY OF MINNESOTA

MinNEAPOLIS, MN 55455 